\title{
Anisotropy in the Compressible Quantum Hall State
}

\author{
Nobuki Maeda \\ Department of Physics, Hokkaido University, Sapporo 060-0810, Japan \\ E-mail: maeda@particle.sci.hokudai.ac.jp
}

\begin{abstract}
Using a mean field theory on the von Neumann lattice, we study compressible anisotropic states around $\nu=l+1 / 2$ in the quantum Hall system. The Hartree-Fock energy of the UCDW are calculated self-consistently. In these states the unidirectional charge density wave (UCDW) seems to be the most plausible state. We show that the UCDW is regarded as a collection of the one-dimensional lattice fermion systems which extend to the uniform direction. The kinetic energy of this one-dimensional system is induced from the Coulomb interaction term and the self-consistent Fermi surface is obtained.
\end{abstract}

\section{Introduction}

As summarized in the table below, the physics at the half-filled $l$-th Landau level is drastically changed in the different Landau level space. In particular, the nature of the anisotropic states discovered in the third or higher Landau levels are unknown until now. At the half-filled lowest and second Landau levels, transition to the anisotropic state was also observed in the presence of the periodic potential or in-plane magnetic field, respectively.

The charge density wave state (CDW) 0 国阳国 is a candidate of the anisotropic state. We study the compressible charge density wave (CCDW) states, which has no energy gap, by the Hartree-Fock approximation using the von Neumann lattice formalism. 6 .

There are two types of The CCDW states, the one is the unidirectional charge density wave (UCDW) state and the other is the compressible Wigner crystal (CWC) state. The UCDW state has a charge density which is uniform in one direction and oscillates in the other direction. The charge density of the CWC state has the same periodicity of the von Neumann lattice. The classification of the CDW is given in the next table.

\begin{tabular}{|c|c|c|}
\hline & Experiment & Theory \\
\hline$l=0$ & Isotropic Fermi liquid & Composite Fermion \\
$l=1$ & FQHE & Paired State (?) \\
$l>1$ & Anisotropic states & UCDW (?) \\
\hline
\end{tabular}




\section{Mean Field Theory on the von Neumann Lattice}

Let us consider the two-dimensional electron system in a perpendicular magnetic field $B$ which is described by a Hamiltonian $H=H_{0}+H_{\text {int }}$,

$$
\begin{aligned}
H_{0} & =\int d^{2} r \psi^{\dagger}(\mathbf{r}) \frac{(\mathbf{p}+e \mathbf{A})^{2}}{2 m} \psi(\mathbf{r}), \\
H_{\mathrm{int}} & =\frac{1}{2} \int d^{2} r d^{2} r^{\prime}: \rho(\mathbf{r}) V\left(\mathbf{r}-\mathbf{r}^{\prime}\right) \rho\left(\mathbf{r}^{\prime}\right): .
\end{aligned}
$$

where $\rho(\mathbf{r})=\psi^{\dagger}(\mathbf{r}) \psi(\mathbf{r}), \nabla \times \mathbf{A}=B$, and $V(\mathbf{r})=q^{2} / \mathrm{r}$.

In the von Neumann lattice formalism, 6 , the electron field is expanded as $\psi(\mathbf{r})=\sum_{l, \mathbf{X}} b_{l}(\mathbf{X}) W_{l, \mathbf{X}}(\mathbf{r})$, where $b$ is an anti-commuting annihilation operator and $\mathbf{X}$ is an integer valued two-dimensional coordinate. The Wannier basis $W_{l, \mathbf{X}}(\mathbf{r})$ 's are orthonormal complete basis in the $l$-th Landau level as $\left(W_{l, \mathbf{X}}(\mathbf{r}), W_{l^{\prime}, \mathbf{X}^{\prime}}(\mathbf{r})\right)=$ $\delta_{l l^{\prime}} \delta_{\mathbf{X}, \mathbf{X}^{\prime}} \cdot W_{l, \mathbf{X}}(\mathbf{r})$ are localized at two-dimensional lattice sites $m \mathbf{e}_{1}+n \mathbf{e}_{2}$ for $\mathbf{X}=$ $(m, n)$, where $\mathbf{e}_{1}=(r a, 0), \mathbf{e}_{2}=(a / r \tan \theta, a / r)$, and $a=\sqrt{2 \pi / e B}$. The area of the unit cell is $\mathbf{e}_{1} \times \mathbf{e}_{2}=a^{2}$. We set $a=1$ in the following calculation.

The Bloch wave basis $u_{l, \mathbf{p}}(\mathbf{r})=\sum_{\mathbf{X}} W_{l, \mathbf{X}}(\mathbf{r}) e^{i \mathbf{p} \cdot \mathbf{X}}$ is another useful basis. We can obtain the charge density profile of the CDW state using this basis. The lattice momentum $\mathbf{p}$ is defined in the Brillouin zone (BZ), $\left|p_{i}\right| \leq \pi$. Using this basis, we obtain another expansion of the electron field as $\psi(\mathbf{r})=\sum_{l} \int_{\mathrm{BZ}} \frac{d^{2} p}{(2 \pi)^{2}} a_{l}(\mathbf{p}) u_{l, \mathbf{p}}(\mathbf{r})$. The Fourier transformed density operator $\tilde{\rho}(\mathbf{k})=\int d^{2} r \rho(\mathbf{r}) e^{i \mathbf{k} \cdot \mathbf{r}}$ is written as

$$
\tilde{\rho}(\tilde{\mathbf{k}})=\sum_{l, l^{\prime}} \int_{\mathrm{BZ}} \frac{d^{2} p}{(2 \pi)^{2}} a_{l}^{\dagger}(\mathbf{p}+\mathbf{k}) M_{l l^{\prime}}(\mathbf{k}) e^{i f(\mathbf{p}+\mathbf{k}, \mathbf{p})} a_{l^{\prime}}(\mathbf{p}),
$$

where $\tilde{\mathbf{k}}=\left(k_{x} / r, r k_{y}-k_{x} / r \tan \theta\right)$. The phase function $f$ is given by $f\left(\mathbf{p}^{\prime}, \mathbf{p}\right)=$ $\int_{\mathbf{p}}^{\mathbf{p}^{\prime}}(\tilde{\mathbf{A}}(\mathbf{p})-\nabla \lambda(\mathbf{p})) \cdot d \mathbf{p}$, where $\tilde{\mathbf{A}}(\mathbf{p})=\left(p_{y} / 2 \pi, 0\right)$, which represents a uniform magnetic field in the momentum space $\nabla_{p} \times \tilde{\mathbf{A}}(\mathbf{p})=-1 / 2 \pi$. The following boundary condition is required, $e^{i \lambda(\mathbf{p}+2 \pi \mathbf{N})-i \lambda(\mathbf{p})}=(-1)^{N_{x}+N_{y}} e^{-i N_{y} p_{x}}, N_{x}, N_{y}$ : integers. The matrix $M_{l, l^{\prime}}$ is given by $\left(\frac{l}{l^{\prime}}\right)^{\frac{1}{2}}\left(\frac{k_{x}+i k_{y}}{\sqrt{4 \pi}}\right)^{l^{\prime}-l} L_{l}^{\left(l^{\prime}-l\right)}\left(\frac{k^{2}}{4 \pi}\right) e^{-\frac{k^{2}}{8 \pi}}$, for $l \leq l^{\prime}$ and $M_{l^{\prime} l}(\mathbf{k})=M_{l l^{\prime}}^{*}(-\mathbf{k})$.

\begin{tabular}{|c|c|c|}
\hline \multicolumn{2}{|c|}{ Charge density wave (CDW) } \\
\hline $\begin{array}{c}\text { Incompressible } \\
\text { Gapfull }\end{array}$ & \multicolumn{2}{|c|}{ Compressible,Gapless } \\
\cline { 2 - 3 } & (CCDW) \\
\cline { 2 - 3 } & $\begin{array}{c}\text { Unidirectional } \\
\text { (UCDW) }\end{array}$ & Wigner crystal \\
& (CWC) \\
\hline
\end{tabular}


The free Hamiltonian $H_{0}$ and interaction Hamiltonian $H_{\text {int }}$ become

$$
\begin{aligned}
H_{0} & =\sum_{l, \mathbf{X}} \omega_{c}\left(l+\frac{1}{2}\right) b_{l}^{\dagger}(\mathbf{X}) b_{l}(\mathbf{X}) \\
H_{\mathrm{int}} & =\frac{1}{2} \sum_{\mathbf{X}_{i}, l_{i}, l_{i}^{\prime}}: b_{l_{1}}^{\dagger}\left(\mathbf{X}_{1}\right) b_{l_{1}^{\prime}}\left(\mathbf{X}_{1}^{\prime}\right) V_{l_{1} l_{1}^{\prime} l_{2} l_{2}}(\mathbf{X}, \mathbf{Y}, \mathbf{Z}) b_{l_{2}}^{\dagger}\left(\mathbf{X}_{2}\right) b_{l_{2}^{\prime}}\left(\mathbf{X}_{2}^{\prime}\right):
\end{aligned}
$$

where $\mathbf{X}=\mathbf{X}_{1}-\mathbf{X}_{1}^{\prime}, \mathbf{Y}=\mathbf{X}_{2}-\mathbf{X}_{2}^{\prime}$, and $\mathbf{Z}=\mathbf{X}_{1}-\mathbf{X}_{2}^{\prime}$. Thus the system is translationally invariant on the lattice. $\tilde{V}(\mathbf{k})=2 \pi q^{2} / k$ for $\mathbf{k} \neq 0$ and $\tilde{V}(0)=0$ due to the charge neutrality condition.

The Hamiltonian $H$ is invariant under the transformation $b_{l}(\mathbf{X}) \rightarrow e^{i \mathbf{K} \cdot \mathbf{X}} b_{l}(\mathbf{X})$, $\lambda(\mathbf{p}) \rightarrow \lambda(\mathbf{p}+\mathbf{K})+K_{x} p_{y}$. This transformation is the magnetic translation in the momentum space $\mathbf{p} \rightarrow \mathbf{p}+\mathbf{K}$. This invariance is referred to as the K-invariance in the composite fermion model.

\section{Hartree-Fock energy for the CCDW states}

We consider only the intra-Landau level's energy of the $l$-th Landau level. The filling factor $\nu$ is written as $\nu=l+\bar{\nu}$. Mean field $U_{l}\left(\mathbf{X}^{\prime}, \mathbf{X}\right)=\left\langle b_{l}^{\dagger}(\mathbf{X}) b_{l}\left(\mathbf{X}^{\prime}\right)\right\rangle$ for the CCDW which has the translational invariance on the von Neumann lattice, that is, $U_{l}\left(\mathbf{X}-\mathbf{X}^{\prime}\right)=U_{l}\left(\mathbf{X}, \mathbf{X}^{\prime}\right), U_{l}(0)=\bar{\nu}$. The Hartree-Fock Hamiltonian in the $l$-th Landau level, then, becomes

$H_{\mathrm{HF}}^{(l)}=\sum_{\mathbf{X}, \mathbf{X}^{\prime}} U_{l}\left(\mathbf{X}-\mathbf{X}^{\prime}\right)\left\{\tilde{v}_{l}\left(2 \pi\left(\hat{\mathbf{X}}-\hat{\mathbf{X}}^{\prime}\right)\right)-v_{l}\left(\hat{\mathbf{X}}-\hat{\mathbf{X}}^{\prime}\right)\right\}\left\{b_{l}^{\dagger}(\mathbf{X}) b_{l}\left(\mathbf{X}^{\prime}\right)-\frac{1}{2} U_{l}\left(\mathbf{X}^{\prime}-\mathbf{X}\right)\right\}$.

where $\tilde{v}_{l}(\mathbf{k})=\left\{L_{l}\left(\frac{k^{2}}{4 \pi}\right)\right\}^{2} e^{-\frac{k^{2}}{4 \pi}} \tilde{V}(\mathbf{k}), v_{l}(\mathbf{X})=\int \frac{d^{2} k}{(2 \pi)^{2}} \tilde{v}_{l}(\mathbf{k}) e^{i \mathbf{k} \cdot \mathbf{X}}$, and $\hat{\mathbf{X}}=(r m+$ $n / r \tan \theta, n / r)$ for $\mathbf{X}=(m, n)$. Thus the continuum system with a magnetic field is transformed to the lattice system without a magnetic field!

The self-consistency equations for the kinetic energy $\varepsilon_{l}$ is given by $\varepsilon_{l}(\mathbf{p}, \bar{\nu})=$ $\int_{\mathrm{BZ}} \frac{d^{2} p^{\prime}}{(2 \pi)^{2}} \tilde{v}_{l}^{\mathrm{HF}}\left(\mathbf{p}^{\prime}-\mathbf{p}\right) \theta\left(\mu_{l}-\varepsilon_{l}\left(\mathbf{p}^{\prime}, \bar{\nu}\right)\right)$, where $\mu_{l}$ is the chemical potential and $\tilde{v}_{l}^{\mathrm{HF}}$ is defined by $\tilde{v}_{l}^{\mathrm{HF}}(\mathbf{p})=\sum_{\mathbf{X}}\left\{\tilde{v}_{l}(2 \pi(\hat{\mathbf{X}}))-v_{l}(\hat{\mathbf{X}})\right\} e^{-i \mathbf{p} \cdot \mathbf{X}}$. The energy per particle in the $l$-th Landau level is given by $E^{(l)}=\frac{1}{2 \bar{\nu}} \sum_{\mathbf{X}}\left|U_{l}(\mathbf{X})\right|^{2}\left\{\tilde{v}_{l}(2 \pi \hat{\mathbf{X}})-v_{l}(\hat{\mathbf{X}})\right\} . E^{(l)}$ is a function of $\bar{\nu}, r$ and $\theta$. The parameters $r$ and $\theta$ are determined so as to minimize the energy $E^{(l)}$ at a fixed $\bar{\nu}$. Existence of a Fermi surface breaks the K-invariance inevitably. There are two types of self-consistent Fermi seas.

(a) Belt-shaped Fermi sea (UCDW state) :

$$
U_{l}(\mathbf{X})=\delta_{m, 0} \frac{\sin \left(p_{\mathrm{F}} n\right)}{\pi n}, p_{\mathrm{F}}=\pi \bar{\nu}
$$

(b) Diamond-shaped Fermi sea (CWC state for $\bar{\nu}=1 / 2)$ :

$$
U_{l}(\mathbf{X})=\frac{2}{(\pi)^{2}} \frac{\sin \frac{\pi}{2}(m+n) \sin \frac{\pi}{2}(m-n)}{m^{2}-n^{2}} .
$$


We calculated the Hartree-Fock energy for (a) and (b) at $l<4$. As a result, we found that the UCDW state is the lowest energy state in all cases. 7 Therefore the UCDW state is the most plausible state in the CCDW states.

Using the mean field of (a), the kinetic term in $H_{\mathrm{HF}}^{(l)}$ is written as

$$
K_{\mathrm{HF}}^{(l)}=\sum_{m} \int \frac{d p_{y}}{2 \pi} a_{l, m}^{\dagger}\left(p_{y}\right) \varepsilon_{l}\left(p_{y}, \bar{\nu}\right) a_{l, m}\left(p_{y}\right)
$$

where $a_{l, m}\left(p_{y}\right)=\sum_{n} b_{l}(\mathbf{X}) e^{-i p_{y} n}$ for $\mathbf{X}=(m, n)$. Therefore the UCDW state is regarded as a collection of the one-dimensional lattice Fermi-gas systems which extend to the y-direction.

Using the Buttiker-Landauer formula, the conductance of the UCDW $\sigma_{x x}=0$, $\sigma_{y y}=n_{x} \frac{e^{2}}{2 \pi}$, where $n_{x}$ is a number of the one-dimensional channels. If we take $\sigma_{x y}=\nu e^{2} / 2 \pi$, the resistance becomes $\rho_{x x}=\frac{n_{x}}{\nu^{2}} \frac{2 \pi}{e^{2}}, \rho_{y y}=0$. Thus the formation of the UCDW leads the anisotropy in the magnetoresistance.

\section{Summary and discussion}

We have studied the CCDW state, which is gapless state and has an anisotropic Fermi surface. We obtained two types of the CCDW state, the UCDW state and CWC state. By calculating the Hartree-Fock energy, the UCDW is found to have a lower energy at the half-filled Landau levels. The UCDW state is regarded as a system which consists of many one-dimensional lattice Fermi-gas systems which extend to the uniform direction. Formation of this structure could be the origin of the anisotropy observed in experiments. Theoretical works to include fluctuations around the mean field solution are necessary. Since there is no energy gap in the CCDW state, the fluctuation effect might be large compared with the gapfull CDW state.

\section{References}

1. A. A. Koulakov, M. M. Fogler, and B. I. Shklovskii, Phys. Rev. Lett. 76, 499 (1996); Phys. Rev. B 54, 1853 (1996).

2. R. Moessner and J. T. Chalker, Phys. Rev. B 54, 5006 (1996).

3. E. Fradkin and S. A. Kivelson, Phys. Rev. B 598065 (1999).

4. E. H. Rezayi, F. D. M. Haldane, and K. Yang, Phys. Rev. Lett. 83, 1219 (1999).

5. T. Jungwirth, A. H. MacDonald, L. Smrčka, and S. M. Girvin, condmat/9905353.

6. K. Ishikawa, N. Maeda, and T. Ochiai, Phys. Rev. Lett. 82, 4292 (1999).

7. N. Maeda, cond-mat/9908373. 